\title{
The construction problem for Hodge numbers modulo an integer in positive characteristic
}

\author{
Remy van Dobben de Bruyn ${ }^{1,2}$ and Matthias Paulsen ${ }^{3}$ \\ ${ }^{1}$ Department of Mathematics, Princeton University, Fine Hall, Washington Road, Princeton, NJ 08544, USA; \\ E-mail: rdobben@math.princeton.edu. \\ ${ }^{2}$ Institute for Advanced Study, 1 Einstein Drive, Princeton, NJ 08540, USA. \\ ${ }^{3}$ Institute of Algebraic Geometry, Gottfried Wilhelm Leibniz Universität Hannover, Welfengarten 1, D-30167 Hannover, \\ Germany; E-mail: paulsen@math.uni-hannover.de.
}

Received: 19 March 2020; Revised: 6 July 2020; Accepted: 15 August 2020

2020 Mathematics Subject Classification: Primary-14F99; Secondary-14G17, 14A10, 14E99

\begin{abstract}
Let $k$ be an algebraically closed field of positive characteristic. For any integer $m \geq 2$, we show that the Hodge numbers of a smooth projective $k$-variety can take on any combination of values modulo $m$, subject only to Serre duality. In particular, there are no non-trivial polynomial relations between the Hodge numbers.
\end{abstract}

\section{Introduction}

The Hodge numbers $h^{p, q}(X)=\operatorname{dim}_{\mathbf{C}} H^{q}\left(X, \Omega_{X}^{p}\right)$ of an $n$-dimensional smooth projective variety $X$ over C satisfy the following conditions:

(1) $h^{0,0}(X)=1$ (connectedness).

(2) $h^{p, q}(X)=h^{n-p, n-q}(X)$ for all $0 \leq p, q \leq n$ (Serre duality).

(3) $h^{p, q}(X)=h^{q, p}(X)$ for all $0 \leq p, q \leq n$ (Hodge symmetry).

Kotschick and Schreieder showed [8, Theorem 1, consequence (2)] that the only linear relations among the Hodge numbers that are satisfied by all smooth projective $\mathbf{C}$-varieties of dimension $n$ are the ones induced by (1), (2), and (3).

In positive characteristic, Hodge symmetry (3) does not always hold [14, Proposition 16], but Serre duality (2) is still true. Van Dobben de Bruyn proved that (1) and (2) are indeed the only universal linear relations among the Hodge numbers of $n$-dimensional smooth projective $k$-varieties if char $k>0$ [5, Theorem 1].

In [11, Theorem 2], Paulsen and Schreieder solved the construction problem over $\mathbf{C}$ for Hodge diamonds modulo an arbitrary integer $m \geq 2$. This means for any dimension $n$ and any collection of integers satisfying the conditions (1), (2), and (3), there exists a smooth projective $\mathbf{C}$-variety of dimension $n$ whose Hodge numbers agree with the given integers modulo $m$. As a corollary, there are no non-trivial polynomial relations among the Hodge numbers, which strengthens the result from [8] on linear relations.

In this paper, we solve the construction problem for Hodge diamonds modulo $m$ in positive characteristic:

(C) The Author(s), 2020. Published by Cambridge University Press. This is an Open Access article, distributed under the terms of the Creative Commons Attribution licence (http://creativecommons.org/licenses/by/4.0/), which permits unrestricted re-use, distribution, and reproduction in any medium, provided the original work is properly cited. 
Theorem 1. Let $k$ be an algebraically closed field of positive characteristic, and let $m \geq 2$ and $n \geq 0$ be integers. Let $\left(a^{p, q}\right)_{0 \leq p, q \leq n}$ be any collection of integers such that $a^{0,0}=1$ and $a^{p, q}=a^{n-p, n-q}$ for all $0 \leq p, q \leq n$. Then there exists a smooth projective $k$-variety $X$ of dimension $n$ such that

$$
h^{p, q}(X) \equiv a^{p, q} \quad(\bmod m)
$$

for all $0 \leq p, q \leq n$.

In analogy to [11, Corollary 3], it follows that there are no polynomial relations among the Hodge numbers in positive characteristic besides (1) and (2) (see Corollary 5.1). This extends the result from [5, Theorem 1] on linear relations.

Theorem 1 also shows that Hodge symmetry may fail arbitrarily badly in positive characteristic. For any dimension $n$ and all $0 \leq p<q \leq n$ with $p+q \neq n$, not only can the Hodge numbers $h^{p, q}$ and $h^{q, p}$ be different, but can even be incongruent modulo any integer $m \geq 2$. Note that Hodge symmetry (3) is a consequence of Serre duality (2) if $p+q=n$ and thus always holds in the middle row of the Hodge diamond.

A complete classification of the possible Hodge diamonds of smooth projective $k$-varieties, i.e. a version of Theorem 1 without the 'modulo $m$ ' part, seems to be very hard already when Hodge symmetry is true; see [13] for strong partial results on this in characteristic zero.

The structure of our proof is similar to [11], with some improvements. First we solve the construction problem modulo $m$ for the outer Hodge numbers, i.e. the Hodge numbers $h^{p, q}$ with $p \in\{0, n\}$ or $q \in\{0, n\}$ (see Proposition 3.1). Then we prove that for any smooth projective $k$-variety, there exists a sequence of blowups in smooth centres such that the inner Hodge numbers of the blowup, i.e. the Hodge numbers $h^{p, q}$ with $1 \leq p, q \leq n-1$, attain any given values in $\mathbf{Z} / m$ satisfying Serre duality (2). Hence we obtain the following result, which might be of independent interest:

Theorem 2. Let $k$ be an algebraically closed field of positive characteristic, and let $m \geq 2$ and $n \geq 0$ be integers. Let $X$ be a smooth projective $k$-variety of dimension $n$, and let $\left(a^{p, q}\right)_{1 \leq p, q \leq n-1}$ be any collection of integers such that $a^{p, q}=a^{n-p, n-q}$ for all $1 \leq p, q \leq n-1$. Then there exists a smooth projective $k$-variety $\tilde{X}$ birational to $X$ such that

$$
h^{p, q}(\tilde{X}) \equiv a^{p, q} \quad(\bmod m)
$$

for all $1 \leq p, q \leq n-1$.

The analogous result in characteristic zero was obtained in [11, Theorem 5]. The fact that all outer Hodge numbers are birational invariants in positive characteristic was proven by Chatzistamatiou and Rülling [3, Theorem 1], so Theorem 2 is the best possible statement. Again, it follows that the result from [5, Theorem 3] on linear birational invariants extends to polynomials (see Corollary 5.2).

In analogy with [5, Theorem 2], our constructions only need Serre's counterexample [14, Proposition 16] to generate all Hodge asymmetry. While the structure of our argument is similar to [11], the absence of condition (3) in positive characteristic raises new difficulties for both the inner and outer Hodge numbers. There is a quick proof of Theorem 2 assuming embedded resolution of singularities in positive characteristic; see Theorem 4.4 The proof we present is similar but does a little more work to avoid using embedded resolution. It relies on Maruyama's theory of elementary transformations of vector bundles, which we briefly recall in the Appendix.

In Section 2, we state and prove some lemmas on Hodge numbers that are used later. The constructions for outer and inner Hodge numbers are carried out in Sections 3 and 4, respectively. Finally, we deduce corollaries on polynomial relations in Section 5.

\section{Notation}

Throughout this paper, we fix an algebraically closed field $k$ of positive characteristic and an integer $m \geq 2$. 


\section{Some lemmas on Hodge numbers}

In this section, we collect some standard results on Hodge numbers that we will use repeatedly in the arguments. The only difference between the situation in characteristic zero [8, 11] and positive characteristic [5] comes from asymmetry of Hodge diamonds; and as in [5], the only example we need is Serre's surface:

Theorem 2.1. There exists a smooth projective $k$-variety $S$ of dimension two such that $h^{1,0}(S)=0$ and $h^{0,1}(S)=1$.

Proof. See [14, Proposition 16], or [5, Proposition 1.4] for a short modern account.

We use the following well-known formula for Hodge numbers under blowups.

Lemma 2.2. Let $X$ be a smooth projective $k$-variety, let $Z \subseteq X$ be a smooth subvariety of codimension $r$, and let $\tilde{X} \rightarrow X$ be the blowup of $X$ at $Z$. Then the Hodge numbers of $\tilde{X}$ satisfy

$$
h^{p, q}(\tilde{X})=h^{p, q}(X)+\sum_{i=1}^{r-1} h^{p-i, q-i}(Z) .
$$

A consequence that will be used repeatedly is that any blowup construction carried out $m$ times does not change the Hodge numbers modulo $m$.

Proof (Lemma 2.2). See for example, [6, Corollary IV.1.1.11]. As noted by Achinger and Zdanowicz [2, Corollary 2.8], it is also an immediate consequence of Voevodsky's motivic blowup formula [15, Proposition 3.5.3] and Chatzistamatiou-Rülling's action of Chow groups on Hodge cohomology [3].

The Hodge numbers of a product $X_{1} \times X_{2}$ can be easily described in terms of the Hodge numbers of $X_{1}$ and $X_{2}$ by a Künneth-type formula.

Lemma 2.3. Let $X_{1}$ and $X_{2}$ be smooth projective $k$-varieties. Then the Hodge numbers of $X:=X_{1} \times X_{2}$ are given by

$$
h^{p, q}(X)=\sum_{\substack{p_{1}+p_{2}=p \\ q_{1}+q_{2}=q}} h^{p_{1}, q_{1}}\left(X_{1}\right) \cdot h^{p_{2}, q_{2}}\left(X_{2}\right) .
$$

Proof. We have $\Omega_{X}=\pi_{1}^{*} \Omega_{X_{1}} \oplus \pi_{2}^{*} \Omega_{X_{2}}$, and thus

$$
\Omega_{X}^{p}=\bigoplus_{p_{1}+p_{2}=p} \pi_{1}^{*} \Omega_{X_{1}}^{p_{1}} \otimes \pi_{2}^{*} \Omega_{X_{2}}^{p_{2}} .
$$

Hence, using the classical Künneth formula for quasi-coherent sheaves, we get

$$
\begin{aligned}
H^{q}\left(X, \Omega_{X}^{p}\right) & =\bigoplus_{p_{1}+p_{2}=p} H^{q}\left(X, \pi_{1}^{*} \Omega_{X_{1}}^{p_{1}} \otimes \pi_{2}^{*} \Omega_{X_{2}}^{p_{2}}\right) \\
& =\bigoplus_{\substack{p_{1}+p_{2}=p \\
q_{1}+q_{2}=q}} H^{q_{1}}\left(X_{1}, \Omega_{X_{1}}^{p_{1}}\right) \otimes H^{q_{2}}\left(X_{2}, \Omega_{X_{2}}^{p_{2}}\right) .
\end{aligned}
$$

The next lemma provides a weak Lefschetz theorem for sufficiently ample hypersurfaces.

Lemma 2.4. Let $X$ be a smooth projective $k$-variety of dimension $n+1$ with a very ample line bundle $\mathscr{L}=\mathcal{O}_{X}(H)$. Let $d_{0} \in \mathbf{Z}_{>0}$ such that $H^{q}\left(X, \Omega_{X}^{p}(-d H)\right)=0$ when $d \geq d_{0}$ and $p+q \leq n$. Then any smooth divisor $Y \in|d H|$ with $d \geq d_{0}$ satisfies $h^{p, q}(Y)=h^{p, q}(X)$ when $p+q \leq n-1$. 
Proof. The short exact sequence

$$
\left.0 \rightarrow \Omega_{X}^{p}(-d H) \rightarrow \Omega_{X}^{p} \rightarrow \Omega_{X}^{p}\right|_{Y} \rightarrow 0
$$

shows that for all $p+q \leq n-1$ and all $e \geq 0$, we have

$$
H^{q}\left(X, \Omega_{X}^{p}(-e H)\right)=H^{q}\left(Y,\left.\Omega_{X}^{p}(-e H)\right|_{Y}\right) .
$$

We will prove by induction on $p$ that $H^{q}\left(Y,\left.\Omega_{X}^{p}(-e H)\right|_{Y}\right)=H^{q}\left(Y, \Omega_{Y}^{p}(-e H)\right)$ for all $e \geq 0$ and $p+q \leq n-1$. Together with (2.1), this proves the result by taking $e=0$. The base case $p=0$ is trivial since $\left.\mathscr{O}_{X}\right|_{Y}=\mathscr{O}_{Y}$. For $p>0$, the inductive hypothesis, (2.1), and the assumption on $d_{0}$ imply

$$
H^{q}\left(Y, \Omega_{Y}^{i}(-e H)\right)=H^{q}\left(Y,\left.\Omega_{X}^{i}(-e H)\right|_{Y}\right)=H^{q}\left(X, \Omega_{X}^{i}(-e H)\right)=0
$$

for $i+q \leq n-1, e \geq d_{0}$, and $i<p$. The conormal sequence

$$
\left.0 \rightarrow \mathcal{O}_{Y}(-Y) \rightarrow \Omega_{X}^{1}\right|_{Y} \rightarrow \Omega_{Y}^{1} \rightarrow 0
$$

gives a short exact sequence

$$
\left.0 \rightarrow \Omega_{Y}^{p-1}(-Y) \rightarrow \Omega_{X}^{p}\right|_{Y} \rightarrow \Omega_{Y}^{p} \rightarrow 0
$$

since $\mathcal{O}_{Y}(-Y)$ is a line bundle. Now (2.2) gives

$$
H^{q}\left(Y, \Omega_{Y}^{p-1}(-Y-e H)\right)=H^{q}\left(Y, \Omega_{Y}^{p-1}(-(d+e) H)\right)=0
$$

for $p+q \leq n$ and $e \geq 0$. Thus, (2.3) shows that the natural map

$$
H^{q}\left(Y,\left.\Omega_{X}^{p}(-e H)\right|_{Y}\right) \rightarrow H^{q}\left(Y, \Omega_{Y}^{p}(-e H)\right)
$$

is an isomorphism for $p+q \leq n-1$ and $e \geq 0$, as claimed.

Corollary 2.5. Let $X$ be a smooth projective $k$-variety of dimension $n+1$ with a very ample line bundle $\mathscr{L}=\mathcal{O}_{X}(H)$. Then any smooth divisor $Y \in|d H|$ with $d \gg 0$ satisfies $h^{p, q}(Y)=h^{p, q}(X)$ when $p+q \leq n-1$.

Proof. By Serre vanishing, there exists $d_{0} \in \mathbf{Z}$ such that $H^{q}\left(X, \Omega_{X}^{p}(-d H)\right)=0$ for all $d \geq d_{0}$ and $q \leq n$. Then Lemma 2.4 gives the result.

Remark 2.6. If char $k=0$, then by Nakano vanishing, we may take $d_{0}=1$ in Lemma 2.4. This recovers the usual proof of weak Lefschetz from Nakano vanishing, although usually the implication goes in the other direction. Similarly, if char $k>0$ and Nakano vanishing holds for $X$, then we may take $d_{0}=1$; but in general already Kodaira vanishing may fail in positive characteristic [12].

For our application, it's useful to have some control over the Euler characteristic of $\mathscr{L}^{-1}$.

Lemma 2.7. Let $X$ be a smooth projective $k$-variety of dimension $n+1$, and let $e \in \mathbf{Z}$. Then, up to modifying $X$ by blowups in smooth centres that do not change its Hodge numbers modulo $m$, we may assume that $X$ admits a very ample line bundle $\mathscr{L}=\mathscr{O}_{X}(H)$ such that $\chi\left(X, \mathscr{L}^{-1}\right) \equiv$ $e(\bmod m)$ and such that any smooth divisor $Y \in|H|$ satisfies $h^{p, q}(Y)=h^{p, q}(X)$ when $p+q \leq n-1$. 
Proof. Let $\pi: \tilde{X} \rightarrow X$ be a blowup in $m$ distinct points $p_{1}, \ldots, p_{m} \in X$. Then the blowup formula for Hodge numbers (Lemma 2.2) gives $h^{p, q}(\tilde{X}) \equiv h^{p, q}(X)(\bmod m)$. Let $E_{i}=\pi^{-1}\left(p_{i}\right)$ be the exceptional divisors, and for $r \in\{0, \ldots, m\}$, write $E_{\leq r}=E_{1}+\ldots+E_{r}$. Then the short exact sequence

$$
0 \rightarrow \mathcal{O}_{\tilde{X}}\left(-E_{\leq r}\right) \rightarrow \mathcal{O}_{\tilde{X}} \rightarrow \mathcal{O}_{E_{\leq r}} \rightarrow 0
$$

shows that

$$
\chi\left(\tilde{X}, \mathcal{O}_{\tilde{X}}\left(-E_{\leq r}\right)\right)=\chi\left(\tilde{X}, \mathcal{O}_{\tilde{X}}\right)-\sum_{i=1}^{r} \chi\left(E_{i}, \mathcal{O}_{E_{i}}\right)=\chi\left(X, \mathcal{O}_{X}\right)-r
$$

Take $r \in\{0, \ldots, m-1\}$ with $r \equiv \chi\left(X, \mathcal{O}_{X}\right)-e(\bmod m)$.

Let $\mathscr{M}$ be an ample line bundle on $\tilde{X}$. By Serre vanishing, there exists $a_{0} \in \mathbf{Z}$ such that for all $a \geq a_{0}$, the line bundle $\mathscr{L}=\mathscr{M}^{\otimes a} \otimes \mathcal{O}_{\tilde{X}}\left(E_{\leq r}\right)$ is very ample and satisfies

$$
H^{q}\left(X, \Omega_{X}^{p} \otimes \mathscr{L}^{-d}\right)=0
$$

for $d>0$ and $q \leq n$. Taking $a$ divisible by the product of $m$ and the denominators of the coefficients of the Hilbert polynomial $P(t)=\chi\left(\tilde{X}, \mathscr{M}^{\otimes t} \otimes \mathcal{O}_{\tilde{X}}\left(-E_{\leq r}\right)\right)$, we see that

$$
\chi\left(\tilde{X}, \mathscr{L}^{-1}\right) \equiv \chi\left(\tilde{X}, \mathcal{O}_{\tilde{X}}\left(-E_{\leq r}\right)\right) \equiv e \quad(\bmod m) .
$$

Finally, $\mathscr{L}$ satisfies weak Lefschetz by (2.4) and Lemma 2.4.

\section{Outer Hodge numbers}

In this section, we solve the construction problem for the outer Hodge numbers. Because of Serre duality and the fact that $h^{0,0}=1$, it suffices to consider the Hodge numbers $h^{p, q}$ with $(p, q) \in J_{n}$, where

$$
J_{n}=\{(1,0), \ldots,(n, 0),(0,1), \ldots,(0, n)\}
$$

The main result of this section is the following:

Proposition 3.1. Let $n \geq 0$. For any given integers $a^{1,0}, \ldots, a^{n, 0}$ and $a^{0,1}, \ldots, a^{0, n}$ with $a^{n, 0}=a^{0, n}$, there exists a smooth projective $k$-variety $X$ of dimension $n$ such that

$$
h^{p, q}(X) \equiv a^{p, q} \quad(\bmod m)
$$

for all $(p, q) \in J_{n}$.

The construction will be carried out by induction on the dimension using the weak Lefschetz results from Corollary 2.5 and Lemma 2.7.

Lemma 3.2. Let $n, d \geq 0$ be integers such that $d \geq n-1$. If Proposition 3.1 holds in dimension $d$ for $a^{1,0}, \ldots, a^{d, 0}$ and $a^{0,1}, \ldots, a^{0, d}$ with $a^{d, 0}=a^{0, d}$, then it also holds in dimension $n$ for $a^{1,0}, \ldots, a^{n-1,0}, b$ and $a^{0,1}, \ldots, a^{0, n-1}, b$ for any $b \in \mathbf{Z}$.

Proof. Let $X$ be a smooth projective $k$-variety of dimension $d$ with the given Hodge numbers $a^{p, q}$. We may assume that $d \geq n+1$ by multiplying $X$ with $\mathbf{P}^{2}$, which does not change its outer Hodge numbers in degree $\leq n-1$. By repeatedly replacing $X$ with a smooth hyperplane section of sufficiently high degree, we may further assume that $d=n+1$ by Corollary 2.5. By Lemma 2.7, after possibly replacing $X$ with a blowup that does not change its Hodge numbers modulo $m$, there exists a very ample line bundle $\mathscr{L}$ on $X$ such that 


$$
\chi\left(X, \mathscr{L}^{-1}\right) \equiv(-1)^{n}\left(a^{0, n}-a^{0, n+1}-b\right) \quad(\bmod m)
$$

and such that a smooth section $Y$ of $\mathscr{L}$ satisfies $h^{p, q}(Y) \equiv a^{p, q}(\bmod m)$ for $p+q \leq n-1$. The short exact sequence

$$
0 \rightarrow \mathscr{L}^{-1} \rightarrow \mathcal{O}_{X} \rightarrow \mathcal{O}_{Y} \rightarrow 0
$$

gives $\chi\left(X, \mathscr{L}^{-1}\right)=\chi\left(X, \mathcal{O}_{X}\right)-\chi\left(Y, \mathcal{O}_{Y}\right)$. Since $h^{0, q}(X)=h^{0, q}(Y)$ for $q \leq n-1$, we conclude that

$$
\begin{aligned}
\chi\left(X, \mathscr{L}^{-1}\right) & =(-1)^{n} h^{0, n}(X)+(-1)^{n+1} h^{0, n+1}(X)-(-1)^{n} h^{0, n}(Y) \\
& \equiv(-1)^{n}\left(a^{0, n}-a^{0, n+1}-h^{0, n}(Y)\right) \quad(\bmod m) .
\end{aligned}
$$

With (3.1), we get $h^{0, n}(Y) \equiv b(\bmod m)$, so Serre duality gives $h^{n, 0}(Y) \equiv b(\bmod m)$.

Note that in characteristic zero, Lemma 3.2 immediately implies Proposition 3.1, giving an alternative approach to a variant of [11, Proposition 4]. In positive characteristic, however, the failure of Hodge symmetry raises new difficulties, since e.g. $h^{n-1,0}=h^{0, n-1}$ is true for varieties of dimension $n-1$ but not for all varieties of dimension $n$. This problem is solved in the following construction, which together with Lemma 3.2 implies Proposition 3.1.

Lemma 3.3. Let $n \geq 2$. For any given integers $a^{0,1}, \ldots, a^{0, n-1}$ and $a^{1,0}, \ldots, a^{n-1,0}$, there exists $a$ smooth projective $k$-variety $X$ of dimension $\geq n-1$ such that

$$
h^{p, q}(X) \equiv a^{p, q} \quad(\bmod m)
$$

for all $(p, q) \in J_{n-1}$.

Note that we do not assume $a^{0, n-1}=a^{n-1,0}$ here, so we typically need $\operatorname{dim} X \geq n$.

Proof (Lemma 3.3). First consider the case $n=2$. Let $E$ be an elliptic curve, and let $S$ be the surface from Theorem 2.1. Choose $i \geq 0$ and $j \geq 1$ with $i \equiv a^{0,1}-a^{1,0}(\bmod m)$ and $j \equiv a^{1,0}(\bmod m)$, and set $X=S^{i} \times E^{j}$. Then it follows from Künneth's formula (Lemma 2.3) that $h^{0,1}(X) \equiv i+j \equiv a^{0,1}$ $(\bmod m)$ and $h^{1,0}(X) \equiv j \equiv a^{1,0}(\bmod m)$.

Now assume $n \geq 3$. By Lemma 3.2, we may assume inductively that Proposition 3.1 holds in dimensions $\leq n-1$. Therefore, there exists a smooth projective variety $Y$ of dimension $n-1$ with outer Hodge numbers

$$
h^{p, q}(Y) \equiv\left\{\begin{array}{ll}
(-1)^{q}, & p=0,0 \leq q<n-1 \\
0, & p=0, q=n-1, \\
0, & p>0, q=0
\end{array} \quad(\bmod m)\right.
$$

By Proposition 3.1 in dimension 2, there exists a smooth projective surface $S$ with outer Hodge numbers $h^{1,0}(S) \equiv h^{2,0}(S) \equiv h^{0,2}(S) \equiv 0(\bmod m)$ and $h^{0,1}(S) \equiv 1(\bmod m)$. The Künneth formula from Lemma 2.3 shows that $S \times Y$ has outer Hodge numbers $h^{p, q}(S \times Y) \equiv 0(\bmod m)$ for $(p, q) \in J_{n-1}$, except $h^{0,0}(S \times Y)=1$ and $h^{0, n-1}(S \times Y) \equiv(-1)^{n}(\bmod m)$.

Finally, by Proposition 3.1 in dimension $n-1$, there exists a smooth projective variety $Z$ with outer Hodge numbers given by

$$
h^{p, q}(Z) \equiv\left\{\begin{array}{ll}
a^{p, q}, & (p, q) \in J_{n-1} \backslash\{(0, n-1)\}, \\
a^{n-1,0}, & (p, q)=(0, n-1) .
\end{array} \quad(\bmod m)\right.
$$


Taking $X=Z \times(S \times Y)^{i}$ for $i \geq 0$ gives outer Hodge numbers

$$
h^{p, q}(X) \equiv\left\{\begin{array}{lll}
a^{p, q}, & (p, q) \in J_{n-1} \backslash\{(0, n-1)\}, & (\bmod m) \\
a^{n-1,0}+(-1)^{n} i, & (p, q)=(0, n-1) .
\end{array}\right.
$$

The result follows by taking $i \equiv(-1)^{n}\left(a^{0, n-1}-a^{n-1,0}\right)(\bmod m)$.

\section{Inner Hodge numbers}

The aim of this section is to prove Theorem 2, i.e. to modify the inner Hodge numbers of a smooth projective $k$-variety via successive blowups. We first show how to produce certain subvarieties with asymmetric Hodge numbers that we will blow up later.

Lemma 4.1. Let $X$ be a smooth projective $k$-variety of dimension $n$, let $b, c \in \mathbf{Z}$ be integers, and let $d \in\{2, \ldots, n-2\}$. Then there exists a smooth projective variety $\tilde{X}$ and a birational morphism $\tilde{X} \rightarrow X$ obtained as a composition of blowups in smooth centres that does not change the Hodge numbers modulo $m$ such that $\tilde{X}$ contains a smooth subvariety $W$ of dimension d satisfying

$$
h^{d, 0}(W)=h^{0, d}(W) \equiv 0 \quad(\bmod m)
$$

and

$$
h^{d-1,0}(W) \equiv b, \quad h^{0, d-1}(W) \equiv c \quad(\bmod m) .
$$

Proof. Let $X_{1} \rightarrow X$ be the blowup of $X$ in a point. The assumption on $d$ implies $n \geq 4$, so the exceptional divisor of $X_{1}$ contains $\mathbf{P}^{3}$. By Proposition 3.1, there exists a smooth projective surface $S_{0}$ such that $h^{2,0}\left(S_{0}\right)=h^{0,2}\left(S_{0}\right) \equiv 0(\bmod m)$ and

$$
h^{1,0}\left(S_{0}\right) \equiv b, \quad h^{0,1}\left(S_{0}\right) \equiv c \quad(\bmod m) .
$$

Choose a possibly singular surface $S_{1} \subseteq \mathbf{P}^{3}$ birational to $S_{0}$. By embedded resolution of surfaces [1, Theorem 9.1.3] (see also [4, Theorem 1.2]), there exists a birational morphism $X_{2} \rightarrow X_{1}$ obtained as a composition of blowups in smooth centres contained in $\mathbf{P}^{3}$ such that the strict transform $S$ of $S_{1}$ is smooth. Since $S$ is also birational to $S_{0}$, we have $h^{2,0}(S)=h^{0,2}(S) \equiv 0(\bmod m)$ and

$$
h^{1,0}(S) \equiv b, \quad h^{0,1}(S) \equiv c \quad(\bmod m) .
$$

Consider the blowup $X_{3} \rightarrow X_{2}$ in $S$. The exceptional divisor is a $\mathbf{P}^{n-3}$-bundle $\mathbf{P}_{S}(\mathscr{E})$ over $S$. Let $Z \subseteq \mathbf{P}^{n-3}$ be a smooth hypersurface of degree $d$ in a linear subspace $\mathbf{P}^{d-1} \subseteq \mathbf{P}^{n-3}$; in particular, $Z$ satisfies $h^{d-2,0}(Z)=h^{0, d-2}(Z)=1$.

By Maruyama's theory of elementary transformations (see Proposition A.8), there exists a diagram

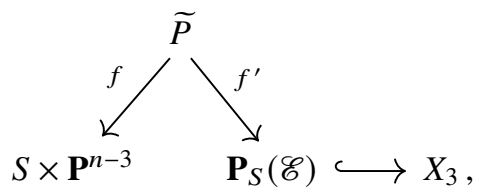

where $f$ and $f^{\prime}$ are blowups in smooth centres $Y$ and $Y^{\prime}$, respectively, such that $Y \cap(S \times Z)$ is smooth. Then the blowup $X_{4} \rightarrow X_{3}$ in $Y^{\prime}$ contains the strict transform

$$
W=\widetilde{S \times Z}=\mathrm{Bl}_{Y \cap(S \times Z)}(S \times Z)
$$

of $S \times Z$ under $f$. Birational invariance of outer Hodge numbers (in the case of a blowup, this is Lemma 2.2) and the Künneth formula (Lemma 2.3) give] 


$$
\begin{aligned}
& h^{d, 0}(W)=h^{0, d}(W)=h^{d, 0}(S \times Z)=h^{2,0}(S) h^{d-2,0}(Z) \equiv 0 \quad(\bmod m), \\
& h^{d-1,0}(W)=h^{d-1,0}(S \times Z)=h^{2,0}(S) h^{d-3,0}(Z)+h^{1,0}(S) h^{d-2,0}(Z) \equiv b \quad(\bmod m) \text {, } \\
& h^{0, d-1}(W)=h^{0, d-1}(S \times Z)=h^{0,2}(S) h^{0, d-3}(Z)+h^{0,1}(S) h^{0, d-2}(Z) \equiv c \quad(\bmod m) .
\end{aligned}
$$

Blowing up $m-1$ more points coming from $X$ and repeating the above construction $m-1$ more times in each exceptional $\mathbf{P}^{n-1}$ separately, the blowup formula of Lemma 2.2 shows that the Hodge numbers of $X$ do not change modulo $m$.

Corollary 4.2. Let $X$ be a smooth projective $k$-variety of dimension $n$, let $b, c \in \mathbf{Z}$ be integers, and let $r \in\{1, \ldots, n-1\}$. Assume that $b=c$ if $r=1$ or $r=n-1$. Then there exists a birational morphism $\tilde{X} \rightarrow X$ obtained by a sequence of blowups in smooth centres such that

$$
h^{r, 1}(\tilde{X}) \equiv h^{r, 1}(X)+b, \quad h^{1, r}(\tilde{X}) \equiv h^{1, r}(X)+c \quad(\bmod m)
$$

and

$$
h^{p, 1}(\tilde{X}) \equiv h^{p, 1}(X), \quad h^{1, p}(\tilde{X}) \equiv h^{1, p}(X) \quad(\bmod m)
$$

for all $p>r$.

Proof. If $r \in\{2, \ldots, n-2\}$, then Lemma 4.1 shows that there exists a successive blowup $X^{\prime} \rightarrow X$ that does not change the Hodge numbers modulo $m$ such that $X^{\prime}$ contains a subvariety $W$ of dimension $r$ satisfying (4.1) and (4.2). Letting $\tilde{X} \rightarrow X^{\prime}$ be the blowup in $W$ gives the result by Lemma 2.2.

For $r=1$, we consider the blowup in $i \geq 0$ points where $i \equiv b=c(\bmod m)$. Then the statement follows again from Lemma 2.2.

For $r=n-1$, we first blow up $X$ in $i \geq 0$ points, where $i \equiv b=c(\bmod m)$. Then, in each exceptional $\mathbf{P}^{n-1}$, we blow up a smooth hypersurface $Z$ of degree $n$. Since $h^{n-2,0}(Z)=h^{0, n-2}(Z)=1$, the result follows from Lemma 2.2.

We are now able to solve the construction problem modulo $m$ for the second outer Hodge numbers, i.e. the inner Hodge numbers $h^{p, q}$ with $p \in\{1, n-1\}$ or $q \in\{1, n-1\}$, via repeated blowups in smooth centres. By Serre duality, it is enough to consider the Hodge numbers $h^{p, q}$ with $(p, q) \in I_{n}$, where

$$
I_{n}=\{(1, q) \mid q \in\{1, \ldots, n-1\}\} \cup\{(p, 1) \mid p \in\{1, \ldots, n-1\}\} .
$$

Corollary 4.3. Let $X$ be a smooth projective $k$-variety of dimension $n$. For any given collection of integers $\left(a^{p, q}\right)_{(p, q) \in I_{n}}$ with $a^{n-1,1}=a^{1, n-1}$, there exists a birational morphism $\tilde{X} \rightarrow X$ obtained by $a$ sequence of blowups in smooth centres such that

$$
h^{p, q}(\tilde{X}) \equiv a^{p, q} \quad(\bmod m)
$$

for all $(p, q) \in I_{n}$.

Proof. For $r \in\{1, \ldots, n-1\}$, let $b=a^{r, 1}-h^{r, 1}(X)$ and $c=a^{1, r}-h^{1, r}(X)$. We see that $b=c$ if $r=1$ or $r=n-1$. Hence, we may apply Corollary 4.2 for all $r \in\{1, \ldots, n-1\}$ in descending order to obtain the result.

Finally, we are ready to prove Theorem 2, which together with Proposition 3.1 implies our main result Theorem 1.

Proof (Theorem 2). We will proceed by induction on $n$. The case $n \leq 1$ is vacuous, as there are no inner Hodge numbers. Let $n \geq 2$, and assume the result is known in all dimensions $\leq n-1$. By Corollary 4.3, 
there exists a birational morphism $X_{1} \rightarrow X$ obtained by a sequence of blowups in smooth centres such that for $(p, q) \in I_{n}$, we have

$$
h^{p, q}\left(X_{1}\right) \equiv a^{p, q}-h^{p-1, q-1}\left(\mathbf{P}^{n-2}\right) \quad(\bmod m) .
$$

Let $X_{2} \rightarrow X_{1}$ be the blowup in a point, and let $\mathbf{P}^{n-2} \subseteq X_{2}$ be a hyperplane in the exceptional divisor. By the induction hypothesis, there exists a birational morphism $\tilde{P} \rightarrow \mathbf{P}^{n-2}$ obtained by a sequence of blowups in smooth centres such that the Hodge numbers of $\tilde{P}$ are given by

$$
h^{p, q}(\tilde{P}) \equiv\left\{\begin{array}{ll}
h^{p, q}\left(\mathbf{P}^{n-2}\right), & p \in\{0, n-2\} \text { or } q \in\{0, n-2\}, \\
a^{p+1, q+1}-h^{p+1, q+1}\left(X_{1}\right), & \text { else. }
\end{array} \quad(\bmod m)\right.
$$

Since $\tilde{P} \rightarrow \mathbf{P}^{n-2}$ is a sequence of blowups in smooth centres, we can blow up the (strict transforms of) the same centres in $X_{2}$ to get a birational morphism $X_{3} \rightarrow X_{2}$ such that the strict transform of $\mathbf{P}^{n-2}$ is $\tilde{P}$. Blowing up $m-1$ more points coming from $X_{1}$ and applying the same construction in each of the exceptional divisors separately gives a birational morphism $X_{4} \rightarrow X_{1}$ that does not change the Hodge numbers modulo $m$ by the blowup formula of Lemma 2.2. Finally, if we let $\tilde{X} \rightarrow X_{4}$ be the blowup in one of the $\tilde{P}$ obtained in this way, we get

$$
h^{p, q}(\tilde{X})=h^{p, q}\left(X_{1}\right)+h^{p-1, q-1}(\tilde{P}) \equiv a^{p, q} \quad(\bmod m)
$$

for all $(p, q)$ with $1 \leq p, q \leq n-1$, which finishes the induction step.

Theorem 4.4. The proof above can be simplified if one assumes embedded resolution of singularities in arbitrary dimension. Indeed, by blowing up a finite number of points, we may assume that $h^{1,1}(X) \equiv a^{1,1}-1(\bmod m)$ and $X$ contains $\mathbf{P}^{n-1}$. Now we claim that we can construct an $(n-2)$ dimensional subvariety $Y$ in a blowup $X^{\prime} \rightarrow X$ with $h^{p, q}\left(X^{\prime}\right) \equiv h^{p, q}(X)(\bmod m)$ such that $h^{p, q}(Y) \equiv a^{p+1, q+1}-h^{p+1, q+1}(X)(\bmod m)$. Then the blowup $\tilde{X} \rightarrow X^{\prime}$ in $Y$ has the required Hodge numbers.

To construct $Y$, first construct any smooth projective variety $Z$ of dimension $n-2$ with the correct outer Hodge numbers using Proposition 3.1. Then $Z$ is birational to a (possibly singular) hypersurface $Z^{\prime} \subseteq \mathbf{P}^{n-1}$. Embedded resolution of $Z^{\prime} \subseteq \mathbf{P}^{n-1}$ gives a birational map $X^{\prime} \rightarrow X$ such that the strict transform of $Z^{\prime}$ is smooth, so $Z^{\prime}$ has the desired outer Hodge numbers by [3, Theorem 1]. By the induction hypothesis, we may blow up further to get the inner Hodge numbers we want. Repeating this construction $m-1$ more times, as usual, gives $h^{p, q}\left(X^{\prime}\right) \equiv h^{p, q}(X)(\bmod m)$.

However, because resolution of singularities is currently unknown in positive characteristic beyond dimension 3, we have developed the above approach using embedded resolution of surfaces, Maruyama's theory of elementary transformations of projective bundles, and the fortuitous fact that the failure of Hodge symmetry is 'generated' by surfaces (see also [5, Theorem 2]).

Remark 4.5. Both the proof of Theorem 2 above (replacing Lemma 4.1 with an easy case of [11, Lemma 6]) and the alternative argument of Theorem 4.4 using resolution of singularities give new methods to prove the characteristic zero result [11, Theorem 5].

Conversely, it is possible to adapt the methods of $[11, \S 3]$ to prove Theorem 2 , using the subvarieties from [11, Lemma 6] as well as projective bundles over the subvarieties from Lemma 4.1, but the analysis is a bit more intricate.

\section{Polynomial relations}

Corollary 5.1. There are no polynomial relations among the Hodge numbers of smooth projective $k$ varieties of the same dimension besides the ones induced by Serre duality. 
Proof. Using [11, Lemma 8], this follows from Theorem 1 in the same way as [11, Corollary 3], except that we now consider the Hodge numbers $h^{p, q}$ with $0 \leq p \leq q \leq n$ and $(p, q) \neq(0,0),(n, n)$.

Corollary 5.2. There are no polynomial relations among the inner Hodge numbers of smooth projective $k$-varieties of any fixed birational equivalence class besides the ones induced by Serre duality.

Proof. This follows from Theorem 2 in a similar fashion.

\section{Appendix. Elementary transformations of vector bundles}

We include a quick coordinate-free proof of Maruyama's theory of elementary transformations of vector bundles [9, 10]. See Theorem A.5 for the main result and Proposition A.8 for the example we will use.

Setup A.1. Let $S$ be a scheme and $D \subseteq S$ a Cartier divisor. We will consider a vector bundle $\mathscr{E}$ on $S$ together with a quotient bundle $\left.\mathscr{E}\right|_{D} \rightarrow \mathscr{F}$. Write $\mathscr{E}^{\prime}$ and $\mathscr{F}^{\prime}$ for the kernels of $\mathscr{E} \rightarrow \mathscr{F}$ and $\left.\mathscr{E}\right|_{D} \rightarrow \mathscr{F}$, respectively, so we get a commutative diagram:

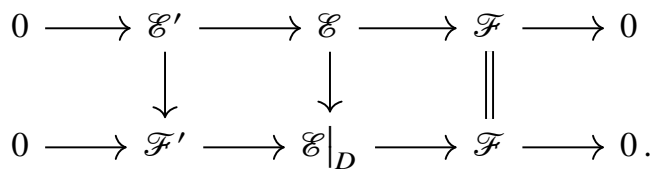

Write $\pi: X=\mathbf{P}_{S}(\mathscr{E}) \rightarrow S$, with tautological quotient line bundle $\pi * \mathscr{E} \rightarrow \mathcal{O}_{\pi}(1)$. The surjection $\mathscr{E} \rightarrow \mathscr{F}$ induces a closed immersion $i: Y=\mathbf{P}_{D}(\mathscr{F}) \hookrightarrow \mathbf{P}_{S}(\mathscr{E})$. Let $f: \tilde{X} \rightarrow X$ be the blowup of $X$ in $Y$ with exceptional divisor $E=f^{-1}(Y)$, and set $\tilde{\pi}=\pi \circ f$. The preimage $\tilde{\pi}^{-1}(D)$ consists of $E$ and $\widetilde{X_{D}}$, whose intersection is the exceptional divisor of $\widetilde{X_{D}} \rightarrow X_{D}=\mathbf{P}_{D}\left(\left.\mathscr{E}\right|_{D}\right)$.

Lemma A.2. Let $X, D$, and $\mathscr{E} \rightarrow \mathscr{F}$ be as in Setup A.1. Then $Y \subseteq X$ is cut out by the image of the composite map

$$
\left(\pi^{*} \mathscr{E}^{\prime}\right) \otimes \mathcal{O}_{\pi}(-1) \rightarrow\left(\pi^{*} \mathscr{E}\right) \otimes \mathcal{O}_{\pi}(-1) \rightarrow \mathcal{O}_{X}
$$

Proof. The map $Y \hookrightarrow X$ is given by applying $\mathbf{P r o j}_{S}$ to the surjection of $\mathcal{O}_{S}$-algebras

$$
\operatorname{Sym}_{\mathscr{O}_{S}}^{*} \mathscr{E} \rightarrow \operatorname{Sym}_{\mathscr{O}_{D}}^{*} \mathscr{F}
$$

The quotient of $\mathbf{S y m}_{\mathscr{O}_{S}}^{*} \mathscr{E}$ by the ideal generated by $\mathscr{E}^{\prime}(-1) \subseteq \mathbf{S y m}_{\mathscr{O}_{S}}^{*} \mathscr{E}$ is $\mathbf{S y m}_{\mathscr{O}_{S}}^{*} \mathscr{F}$, which coincides with $\mathbf{S y m}_{\mathscr{O}_{D}}^{*} \mathscr{F}$ in all positive degrees since $\mathscr{F}$ is supported on $D$. The result follows since a morphism of graded algebras that is eventually an isomorphism induces an isomorphism on Proj.

Corollary A.3. Let $X, D$, and $\mathscr{E} \rightarrow \mathscr{F}$ be as in Setup A.1, and let $q: T \rightarrow S$ be a morphism of schemes. Then morphisms $T \rightarrow \tilde{X}$ of $S$-schemes correspond to pairs $(\mathscr{L}, \phi)$ of a line bundle $\mathscr{L}$ on $T$ and a surjection $\phi: q^{* \mathscr{E}} \rightarrow \mathscr{L}$, up to isomorphism under $q^{*} \mathscr{E}$, such that the image of the composite map

$$
q^{*} \mathscr{E}^{\prime} \rightarrow q^{*} \mathscr{E} \rightarrow \mathscr{L}
$$

is an invertible subsheaf of $\mathscr{L}$. 
Proof. This follows from the universal properties of projective bundles and blowups.

The basic duality of the situation is captured by the following lemma:

Lemma A.4. Let $S, D$, and $\mathscr{E} \rightarrow \mathscr{F}$ be as in Setup A.1. Then $\mathscr{E}^{\prime}$ (respectively, $\mathscr{F}^{\prime}$ ) is locally free on $S$ (respectively, D), and $\mathscr{E}^{\prime} \rightarrow \mathscr{F}^{\prime}$ is another instance of Setup A.1. Applying this operation twice gives $\mathscr{E}(-D) \rightarrow \mathscr{F}(-D)$.

Proof. Since $\mathscr{F}$ is a quotient bundle of $\left.\mathscr{E}\right|_{D}$, it is clear that $\mathscr{F}^{\prime}$ is locally free on $D$. Moreover, since $\mathscr{F}$ has $\mathscr{T}$ or dimension 1 on $S$, we see that $\mathscr{E}^{\prime}$ is a vector bundle. Applying the snake lemma to (A.1) shows that the kernel of $\mathscr{E}^{\prime} \rightarrow \mathscr{F}^{\prime}$ is $\mathscr{E}(-D)$. Applying $\mathscr{T} o r_{*}^{\mathcal{O}_{S}}\left(-, \mathcal{O}_{D}\right)$ to the first row of (A.1) gives the exact sequence

$$
\left.\left.0 \rightarrow \mathscr{F}(-D) \rightarrow \mathscr{E}^{\prime}\right|_{D} \rightarrow \mathscr{E}\right|_{D} \rightarrow \mathscr{F} \rightarrow 0
$$

which shows that the kernel of $\left.\mathscr{E}^{\prime}\right|_{D} \rightarrow \mathscr{F}^{\prime}$ is $\mathscr{F}(-D)$. We omit the verification that the map $\mathscr{E}(-D) \rightarrow \mathscr{F}(-D)$ is obtained from the original one by twisting with $\mathcal{O}_{S}(-D)$.

In analogy with the notation of Setup A.1, write $\pi^{\prime}: X^{\prime}=\mathbf{P}_{S}\left(\mathscr{E}^{\prime}\right) \rightarrow S$, with closed subscheme $Y^{\prime}=\mathbf{P}_{D}\left(\mathscr{F}^{\prime}\right)$ and blowup $f^{\prime}: \tilde{X}^{\prime} \rightarrow X^{\prime}$ in $Y^{\prime}$ with exceptional divisor $E^{\prime}=f^{\prime-1}\left(Y^{\prime}\right)$. Finally, write $X(-D)=\mathbf{P}_{S}(\mathscr{E}(-D))$, with closed subscheme $Y(-D)=\mathbf{P}_{D}(\mathscr{F}(-D))$ and blowup $\tilde{X}(-D) \rightarrow X(-D)$ in $Y(-D)$. The natural isomorphisms $X(-D) \cong X$ and $Y(-D) \cong Y$ lift to a natural isomorphism $\tilde{X}(-D) \cong \tilde{X}$, described in terms of Corollary A.3 by $(\mathscr{L}, \phi) \mapsto(\mathscr{L}(D), \phi(D))$.

The duality of Lemma A.4 directly implies the main theorem of elementary transformations of vector bundles [9, Theorem 1.1, Theorem 1.3]:

Theorem A.5 (Maruyama). Let $X, D$, and $\mathscr{E} \rightarrow \mathscr{F}$ be as in Setup A.1. Then there is a natural isomorphism of $S$-schemes $\tilde{X} \stackrel{\sim}{\rightarrow} \tilde{X}^{\prime}$.

Proof. For an $S$-scheme $Z$, write $h_{Z}$ for the functor $\operatorname{Hom}_{S}(-, Z)$. We will use the description of Corollary A.3 to show that $h_{\tilde{X}}$ and $h_{\tilde{X}^{\prime}}$ are naturally isomorphic, which implies the result by the Yoneda lemma. Let $q: T \rightarrow S$ be a morphism of schemes. Given $(\mathscr{L}, \phi) \in h_{\tilde{X}}(T)$, define $\left(\mathscr{L}^{\prime}, \phi^{\prime}\right) \in h_{\tilde{X}^{\prime}}(T)$ as the image

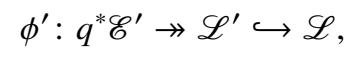

noting that $\mathscr{L}^{\prime}$ is invertible by Corollary A.3. This gives a map $h_{\tilde{X}} \rightarrow h_{\tilde{X}^{\prime}}$, and switching the roles of $\mathscr{E}$ and $\mathscr{E}^{\prime}$ using Lemma A.4 and the natural isomorphism $\tilde{X}(-D) \cong \tilde{X}$ gives the opposite map. The composition takes $(\mathscr{L}, \phi)$ to $(\mathscr{L}(-D), \phi(-D))$, hence, under the identification $\tilde{X}(-D) \cong \tilde{X}$ gives the identity map. The other composition follows dually.

This gives the geometric definition of elementary transformations:

Definition A.6. Let $S$ be a smooth variety, and let $\mathscr{E}$ and $\mathscr{E}^{\prime}$ be vector bundles of the same rank on $S$. We say that there exists an elementary transformation between $X=\mathbf{P}_{S}(\mathscr{E})$ and $X^{\prime}=\mathbf{P}_{S}\left(\mathscr{E}^{\prime}\right)$ if there exists a smooth divisor $D \subseteq S$, a line bundle $\mathscr{L}$ on $S$, and a quotient bundle $\left.\mathscr{E}\right|_{D} \rightarrow \mathscr{F}$ on $D$ such that the kernel of $\mathscr{E} \rightarrow \mathscr{F}$ is $\mathscr{E}^{\prime} \otimes \mathscr{L}$. In this case, Theorem A.5 gives a diagram

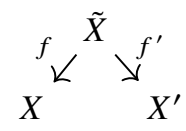

where $f$ and $f^{\prime}$ are blowups in the smooth centres $Y \subseteq X$ and $Y^{\prime} \subseteq X^{\prime}$, respectively.

To construct elementary transformations, we will use the following Bertini smoothness theorem for general sections of a very ample vector bundle. 
Theorem A.7 (Kleiman). Let $X$ be a smooth projective variety of dimension $n$ over a field $k$, let $\mathscr{E}$ be a globally generated vector bundle of rank $r$, and let $\mathscr{L}$ be a very ample line bundle. Then for a general section $\sigma \in \Gamma(X, \mathscr{E} \otimes \mathscr{L})$, the zero locus $Z(\sigma) \subseteq X$ is smooth of codimension $r$ (and nonempty if and only if $r \leq n)$.

As usual, general means that the conclusion holds on a dense Zariski open in the space of sections of $\mathscr{E} \otimes \mathscr{L}$. In particular, there exists a $k$-point when $k$ is infinite.

Proof. See [7, Corollary 3.6 and Remark 3.2(iii)].

This gives the following variant of [9, Theorem 1.12]:

Proposition A.8. Let $S$ be a smooth projective variety of dimension $\leq 2$ over an infinite field $k$, and let $\mathscr{E}$ and $\mathscr{E}^{\prime}$ be vector bundles on $S$ of the same rank $r$. Then there exists an elementary transformation between $X=\mathbf{P}_{S}(\mathscr{E})$ and $X^{\prime}=\mathbf{P}_{S}\left(\mathscr{E}^{\prime}\right)$. Moreover, if $Z_{1}, \ldots, Z_{m} \subseteq X$ are smooth subvarieties, we may assume that $Y \cap Z_{i}$ is smooth for all $i$.

Maruyama's version deals with the case that $\mathscr{E}^{\prime}$ is a trivial bundle, and does not have the final statement. Note that the final statement is not symmetric in $\mathscr{E}$ and $\mathscr{E}^{\prime}$, and we will apply the result when $\mathscr{E}$ is trivial (which is dual to Maruyama's version). Maruyama's result extends to threefolds, which we will not pursue here.

Proof (Proposition A.8). Let $\mathscr{L}$ be a very ample line bundle on $S$. Up to twisting $\mathscr{E}^{\prime}$ by a power of $\mathscr{L}$ and replacing $\mathscr{L}$ by a power, we may assume that $\left(\mathscr{C}^{\prime}\right)^{\vee} \otimes \mathscr{L}^{-1}$ is globally generated and $\pi^{*} \mathscr{L} \otimes \mathscr{O}_{\pi}(1)$ is very ample. By Theorem A.7, there exists a regular section $\sigma$ of $\pi^{*}\left(\mathscr{E}^{\prime}\right)^{\vee} \otimes \mathcal{O}_{\pi}(1)$ such that $Y=Z(\sigma)$ and the $Y \cap Z_{i}$ are smooth.

For each $s \in S$, the intersection $Y \cap X_{S}$ is given by a section of $\mathcal{O}_{\pi}(1)^{r}$ and hence is a linear subspace. Since $\operatorname{dim} S \leq 2$, we have $\operatorname{dim} Y=(\operatorname{dim} S+r-1)-r \leq 1$. If $Y$ contains a vertical line $\ell \subseteq X_{s}$ for some $s \in S$, then $\ell$ is a component of $Y$, and the normal bundle

$$
\mathcal{N}_{\ell / X}=\left.\left(\pi^{*}\left(\mathscr{E}^{\prime}\right)^{\vee} \otimes \mathcal{O}_{\pi}(1)\right)\right|_{\ell}=\mathcal{O}_{\ell}(1)^{r}
$$

has a trivial quotient $\left.\mathcal{N}_{X_{s} / X}\right|_{\ell}=\mathcal{O}_{\ell}^{2}$, which is impossible. Thus, if $D \subseteq S$ is the scheme-theoretic image of $Y$ in $S$, then $D$ is reduced and all fibres of $Y \rightarrow D$ have length 1 , so $Y \rightarrow D$ is an isomorphism. Hence $Y$ corresponds to a section $D \rightarrow \mathbf{P}_{D}\left(\left.\mathscr{E}\right|_{D}\right)$, i.e. a 1-dimensional quotient $\left.\mathscr{E}\right|_{D} \rightarrow \mathscr{F}$. Since $\sigma$ is a regular section, the Koszul complex

$$
0 \rightarrow K_{r} \rightarrow \ldots \rightarrow K_{1} \rightarrow K_{0} \rightarrow \mathcal{O}_{Y} \rightarrow 0
$$

is exact, where $K_{i}=\pi^{*}\left(\bigwedge^{i} \mathscr{E}^{\prime}\right) \otimes \mathcal{O}_{\pi}(-i)$. The projection formula gives

$$
R \pi_{*}\left(K_{i} \otimes \mathcal{O}_{\pi}(1)\right)=\bigwedge^{i} \mathscr{E}^{\prime} \otimes R \pi_{*}\left(\mathcal{O}_{\pi}(-i+1)\right) .
$$

Since $\pi: X \rightarrow S$ is a $\mathbf{P}^{r-1}$-bundle, we have $R \pi_{*}\left(\mathcal{O}_{\pi}(-i+1)\right)=0$ for $1<i \leq r$ and $R^{1} \pi_{*} \mathcal{O}_{X}=0$. Therefore, twisting (A.2) by $\mathcal{O}_{\pi}(1)$ and pushing forward to $S$ gives a short exact sequence

$$
0 \rightarrow \pi_{*}\left(K_{1} \otimes \mathcal{O}_{\pi}(1)\right) \rightarrow \pi_{*}\left(\mathcal{O}_{\pi}(1)\right) \rightarrow \pi_{*}\left(\left.\mathcal{O}_{\pi}(1)\right|_{Y}\right) \rightarrow 0 .
$$

Since $K_{1} \otimes \mathcal{O}_{\pi}(1)=\pi^{*} \mathscr{E}^{\prime}$, this sequence reads

$$
0 \rightarrow \mathscr{E}^{\prime} \rightarrow \mathscr{E} \rightarrow \mathscr{F} \rightarrow 0
$$

so Theorem A.5 gives the desired elementary transformation. 
Acknowledgements. The authors would like to thank Stefan Schreieder for his suggestion to work on this project together. Remy van Dobben de Bruyn thanks Raymond Cheng, Hélène Esnault, and Johan de Jong for useful conversations. The authors are grateful to Jędrzej Garnek for correcting a mistake in the proof of Lemma 2.4 and to the referee for pointing out a problem in an earlier version of Lemma 4.1 as well as other helpful suggestions.

Remy van Dobben de Bruyn is supported by the Oswald Veblen Fund at the Institute for Advanced Study. Matthias Paulsen is supported by the DFG project ‘Topologische Eigenschaften von algebraischen Varietäten' (project no. 416054549). Remy van Dobben de Bruyn thanks the Ludwig-Maximilians-Universität München for their hospitality during a visit where part of this work was carried out.

\section{References}

[1] S. S. Abhyankar, 'Resolution of singularities of embedded algebraic surfaces', Pure and Applied Mathematics 24 (Academic Press, 1966).

[2] P. Achinger and M. Zdanowicz, 'Some elementary examples of non-liftable varieties', Proc. Amer. Math. Soc. 145(11), 4717-4729 (2017), doi: 10.1090/proc/13622

[3] A. Chatzistamatiou and K. Rülling, 'Higher direct images of the structure sheaf in positive characteristic', Algebra Number Theory 5(6), 693-775 (2011). doi: 10.2140/ant.2011.5.693.

[4] S. D. Cutkosky, 'Resolution of singularities for 3-folds in positive characteristic', Amer. J. Math. 131(1), 59-127 (2009), doi:10.1353/ajm.0.0036.

[5] R. van Dobben de Bruyn, 'The Hodge ring of varieties in positive characteristic', in press, 2020, arXiv:2001.02787.

[6] M. Gros, 'Classes de Chern et classes de cycles en cohomologie de Hodge-Witt logarithmique', Mém. Soc. Math. France (N.S.) 21, 1-87 (1985), https://eudml.org/doc/94860.

[7] S. L. Kleiman, 'Geometry on Grassmannians and applications to splitting bundles and smoothing cycles', Inst. Hautes Études Sci. Publ. Math. 36, 281-297 (1969), http://www.numdam.org/item?id=PMIHES_1969_36_281_0.

[8] D. Kotschick and S. Schreieder, 'The Hodge ring of Kähler manifolds', Compos. Math. 149(4), 637-657 (2013), doi:10.1112/S0010437X12000759.

[9] M. Maruyama, 'On a family of algebraic vector bundles', Number Theory, Algebraic Geometry and Commutative Algebra, in honor of Yasuo Akizuki (Kinokuniya Bookstore, 1973), 95-146.

[10] M. Maruyama, 'Elementary transformations in the theory of algebraic vector bundles', Algebraic geometry (La Rábida, 1981); Lecture Notes in Math. 961 (Springer, 1982), 241-266; doi:10.1007/BFb0071286.

[11] M. Paulsen and S. Schreieder, 'The construction problem for Hodge numbers modulo an integer', Algebra Number Theory 13(10), 2427-2434 (2019), doi:10.2140/ant.2019.13.2427.

[12] M. Raynaud, 'Contre-exemple au "vanishing theorem" en caractéristique $p>0$ in C.P. Ramanujam-A Tribute, Tata Inst. Fun. Res. Studies in math., 8 (Springer, 1978), 273-278.

[13] S. Schreieder, 'On the construction problem for Hodge numbers', Geom. Topol. 19(1), 295-342 (2015), doi: 10.2140/gt.2015.19.295.

[14] J.-P. Serre, 'Sur la topologie des variétés algébriques en caractéristique p', in Symposium internacional de topología algebraica (Universidad Nacional Autónoma de México and UNESCO, 1958), 24-53.

[15] V. Voevodsky, Triangulated categories of motives over a field. Cycles, transfers, and motivic homology theories. Ann. of Math. Stud. 143 (Princeton Univ. Press, 2000), 188-238. 